\title{
Work Related Musculoskeletal Disorder amongst Sonologists In Nigeria.
}

\author{
D.E. Bassey ${ }^{1}$, D.U. Eduwem ${ }^{1}$, G.U. Inah ${ }^{1}$, A. Udoaka. ${ }^{2}$, A.O. Akintomide ${ }^{1}$ \\ ${ }^{I}$ Radiology Department, University Of Calabar Teaching Hospital, Cross-River State, Nigeria. \\ ${ }^{2}$ Radiology Department, University Of Uyo Teaching Hospital, Akwa Ibom, Nigeria.
}

\begin{abstract}
Work Related Musculoskeletal Disorder (WRMSD) is now well documented and recognised as an occupational hazard in the diagnostic ultrasound (US) work place. The impact of WRMDS range from minor discomfort to career ending injury. As the US work force in Nigeria increases, more WRMDS are expected.This study aims at documenting the common symptoms of WRMSD amongst cross section of Sonologists in Nigeria and also recommends ways to reduce and prevent this condition. Structured questionnaires distributed to 135 Sonologists were analysed for data relating the symptoms of WRMSD to specific ergonomic factors and other variables. 120 Sonologists met the inclusion criteria of which 117(97.5\%) complained of musculoskeletal (MSk) pains and numbness at various anatomical sites while 3(2.5\%) had no complaints. Analysis shows a positive association between these symptoms and certain US work habits. This study concludes that shoulder pain and finger numbness are the common symptoms of WRMSD in Sonologists in Nigeria. Efforts at reducing and preventing symptoms should focus primarily on educating the Sonologists and Management of institutions on the need to ensure appropriate ergonomic interventions and adaptations.
\end{abstract}

Key Words: Ergonomics, Ultrasound, WRMSD.

\section{Introduction}

WRMSD are disorders of the musculoskeletal system (MSK) and related tissues suspected to be caused by a work place activity (particularly a repetitive activity). For this reason the term Repetitive Strain Injury (RSI) is often used incorrectly to describe WRMSD since not all cases are necessarily caused by repetitive strain of structures of MSK system [1]. However, the World Health Organisation (WHO) in 1985, characterised work related disorders as multi factorial to include physical, organisational, psychological and sociological risk factors. WHO stated that a disorder is work related when work activities and work conditions significantly contribute to their development or exacerbation but are not the sole determinant of causation [2].

Awareness of pain and discomfort associated with the US occupation has been documented since 1985. Many surveys have been done since then resulting in an alarming incidence rate of $90 \%$ [3]. $20 \%$ have been forced to give up their profession as a result of injuries [4].

Unfortunately, technical advances in US have contributed to the increase in WRMSD. Sonographers move rapidly between patients without sufficient rest periods, this result in pain, a sign that muscles are overloaded [5]. The impact of WRMSD ranges from minor discomfort to career ending injury. There are a number of emotional and financial implications that have an impact on the employer and also on quality of patient care. It is therefore important to prevent these injuries [6].

\section{Materials And Method}

The subjects are 135 Sonologists from various health institutions in Nigeria who actively practise sonography and responded to the structured questionnaires. Those with MSk pathology not related to work place activity were excluded. One hundred and twenty (78 males and 42 females, aged 25-65 years) met the inclusion criteria.

Data collected were on specific ergonomic factors such as the type of US room furniture used and equipment specification while the variables include workload, scheduling of US examinations, WRMSD symptoms and treatment.

\section{Results}

$88 \%$ of Sonologists were below 50years while only $12 \%$ were older. (Fig.1). 33(27.5\%) had a work experience of $>10 \mathrm{yrs}$ while $87(72.5 \%)$ were less experienced. Considering workload and schedule, $78(65 \%)$ scanned for an average of $>10 \mathrm{hrs} /$ day while $42(35 \%)$ scanned for shorter duration. $70(58.3 \%)$ scanned an average of 20 patients/day while $50(41.7 \%)$ examined less number of patients. For the US room furniture, $76(63.3 \%)$ scanned on couches above the level of the shoulder, 34(28.4\%) scanned below this level while 10(8.3\%) were not sure. $30(25 \%)$ sat on swivel chairs, $70(58.4 \%)$ on varied types with back support and $20(16.6 \%)$ sat on backless seats. 95 (79.2\%) used units with single monitors while 25 (20.8\%) had double adjustable monitors. 90(75\%) 
Sonologists suffered pains at various sites. $47(52.2 \%)$ felt pains on the right shoulder; lower back pain in 24(26.7\%); neck pain in $10(11.1 \%)$ and $9(10 \%)$ had wrist pain. $27(22.5 \%)$ had numbness on fingers of the right hand while $3(2.5 \%)$ were free of symptoms. (Fig. 2). A total of 117 had WRMSD symptoms. $76(65 \%)$ had relief from the pain on resting or immobilising the affected site while $41(35 \%)$ had to be on medication. For those on medication, only 9 (22\%) sought medical attention and got a prescription while 32(78\%) were on self-medication. No official report was made to the department or the hospital management.

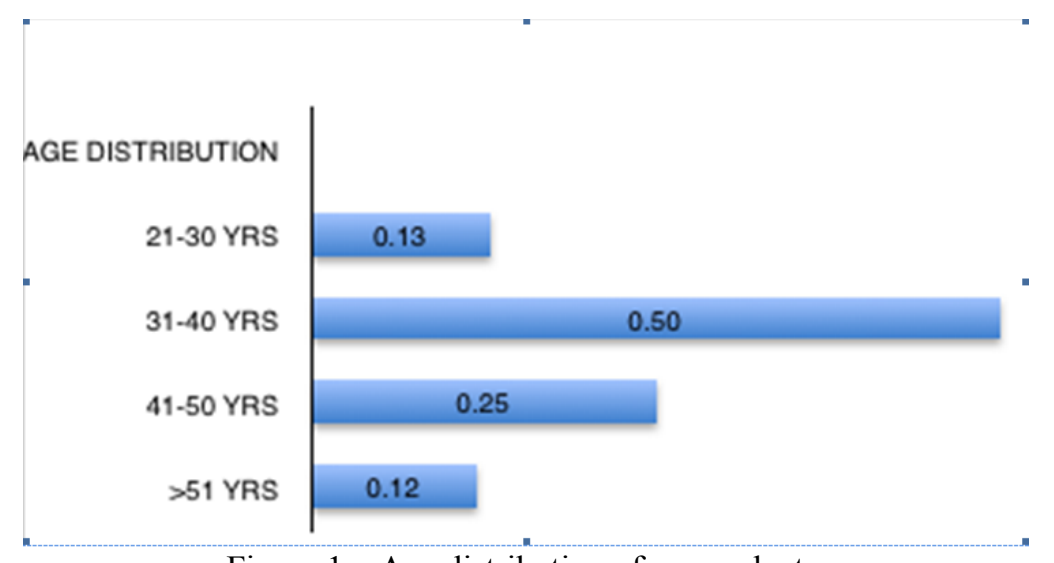

Figure 1. Age distribution of respondents.

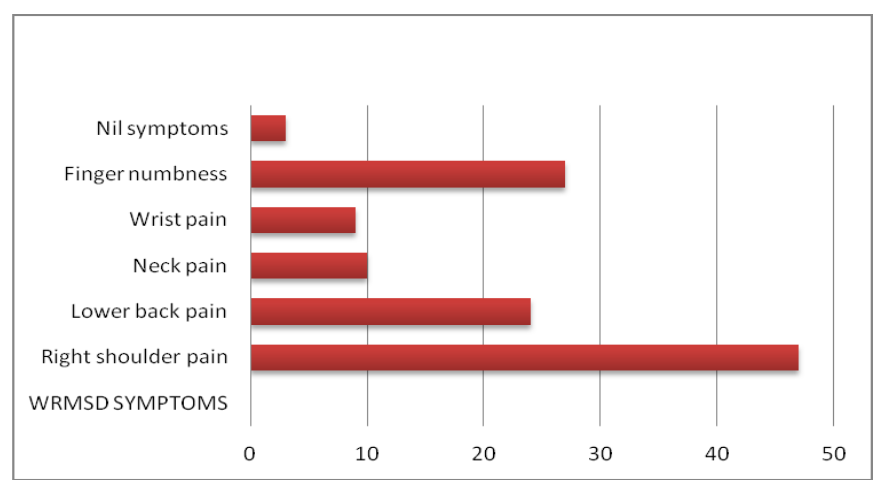

Figure 2. Work related musculoskeletal disorder symptoms.

\section{Discussion}

Bernardino Ramazzim (1633-1714) an Italian Physician and" Father" of occupational/ Industrial medicine was the first to note connections between worker's illnesses and their work environment. He realised that not all workers diseases were attributable to the working environment but could also have been caused by repetition of movement, postural attitudes and anticipated some preventive measures [7]. Oxygen is pumped into muscles and wastes are removed through the normal contraction of muscles during dynamic movement. Static or awkward postures prevent this process, resulting in less oxygen to the muscles and a build up of lactic acid. This is followed by fatigue and eventually pain, a sign that the muscles are over loaded [8].

Across all demographics, shoulder pain is the most common [9]. A similar observation was made in this study in which $(52.2 \%)$ of the Sonologists felt pain in the right shoulder while the others felt pains in other anatomical sites. Shoulder injuries are mostly as a result of excessive abduction of the scanning arm beyond an angle of 30 degrees [3]. Considering specific ergonomic factors such as the height of the examination couch, $29(61.7 \%)$ of those with right shoulder pain scanned on a couch that was above the arm level i.e. arm was above the shoulder level. Abduction of such degree will not only increase activation of the muscle but also take the weight of the transducer \& cord thereby causing more fatigue. The intervention is to reduce arm abduction [5]. Fine motor control of the hand is more precise if abduction of the upper arm is reduced so that most of the hand movement is initiated from the forearm rather than the upper arm [4]. This can be achieved by adjusting the examination table/couch to the appropriate height whose range should be expansive enough to accommodate both standing and seated users. Generally the range is a low height of 22-23 inches and a maximum height of 38inches [10]. An electrically powered height and tilt adjustable (for vascular work) examination table which is also capable of going low enough to allow patients get on and off easily is the best [11]. Cost is a reason that institutions do not provide electronic tables but the cost of WRMDS is considerably more than a table, making the economics of ergonomics worth investigating [3]. Shoulder pain felt by $27.7 \%$ who had the recommended 
height of couch was most probably due to over reaching. The couch needs to be narrow enough so that the sonologist does not have to reach any further than necessary [11].

Pulling the arm across the body to access the controls on the US unit commonly results in shoulder pain to the non-scanning arm [3]. No such symptom was reported in this study. Lower back pain is due to twisting of the trunk and lack of support to the lumbar region. Often using adjustable chairs or stools with back support solves this problem [8]. The chairs need to be mobile and also rotate so that the Sonologist can rotate from the patient to the US unit whilst keeping posture aligned [11]. Only $25 \%$ of the Sonologists sat on swivel chairs that are height and tilt adjustable. Wrist, hand and finger injuries can be caused by many different factors. This study shows that $10 \%$ had wrist pain while $22 \%$ had finger numbness. Nerve damage results from arm abduction, flexion and extension of the wrist and/ or fingers. These motions cause swelling of the soft tissues, which compress \& entrap the nerve of the wrist or finger resulting in pains in the wrist $\&$ feeling of numbness in the fingers $[8,12]$.

The transducer should be of optimum size for an efficient "power grip" (palmer) where the hand could be spread along the wide edge of the transducer rather than a pinch grip and should also be slip resistant to assist grip (textured gloves can help) [13]. Too large a probe is difficult to grasp while too small a transducer requires increased muscular effort, inefficient gripping and pain in the hand [14]. Increasing transducer pressure requires a tighter grip on the transducer and this happens when much pressure is applied with obese patients. To avoid pains in the hand \& wrist resulting from this, standing may be advantageous so that the whole body \& not just the arm can be used to exert the necessary pressure to produce a diagnostic image [3].

Pain in the neck is usually due to incorrect monitor position resulting in twisting \& extending the neck to view the monitor [3]. It is preferable that the monitor is independent of the control panel and the neck flexed between $15-20$ degrees. Adjustments to the height of the chair may be necessary to achieve this. Also installing a monitor at the foot of the exam table for the patients to view will eliminate the need to twist the neck in order to share the monitor with the patient [3]. $11.1 \%$ of the Sonologists in this study had neck pain and most $(79.2 \%)$ had units with single monitors while only (20.8\%) had double monitors.

WRMSD affect a large number of Sonographers/Sonologists particularly those with high workloads and who have been in the profession for a long time [11]. This study shows that despite the work experience of over 10 years, only $18(54.5 \%)$ had symptoms of WRMSD compared to $60(69 \%)$ with less work experience. This is probably due to the fact that the latter are of a younger age group and could take on a higher workload. Reducing the workload by reducing the number of scans may help but the issue is rather the time spent holding the transducer and the ergonomic environment [3]. Scheduling can be arranged such that a variety of exams are done per workday, this decreases strain on MSK tissue specific to one type of exam. It is difficult to specify an allowable limit to the number of exams or patients per day because of the complexity of each diagnostic situation but more exams of shorter duration or fewer of longer duration should be considered [13].

Symptoms of WRMSD should be promptly evaluated by registered health care providers [14]. In this study, only $9(22 \%)$ had prescribed medication but no official report was made to the management of the respective institutions, others were on self-medication. Treatment for WRMSD often has a poor outcome because the affected person goes back to the same environment that initially produced the injury [15].

This study concludes that the common symptoms of WRMSD amongst Sonologists in Nigeria are numbness of the fingers and pain with the right shoulder most affected. Educating the Sonologists on posture and ergonomics are the keys to prevention. Ultrasound units/centres on the other hand need to be informed to ensure appropriate ergonomic interventions and adaptations.

\section{References}

[1]. Work related Musculoskeletal Disorder. A review of the evidences. Commission on behavioural, social sciences and education. The National Academic Press. 1998

[2]. World Health Organisation (WHO). Identification and Control of Work Related Diseases. Technical Report Series. Geneva. 1985. No 714.

[3]. Baker J.P. The "Price" we all pay for ignoring ergonomics in sonography. Society of Radiologists in Ultrasound Newsletter. Jan 2011. Vol. 21. No 1. 3-4

[4]. Pike I, Russo A, Berkowitz J et al. The prevalence of musculoskeletal disorders among diagnostic medical sonographers. Journal of Diagnostic Medical Sonography. Sept-Oct. 1997. Vol. 13-15. 219-227.

[5]. Grieco A, De Vito G et al. Epidemiology of musculoskeletal disorders due to biochemical overloads. Ergonomics. Sept 1998. Vol. 41; 9. 1253-1260.

[6]. Hawkins J, Survey seeks to quantify Technologists. Advance for Imaging and radiation therapy professional. July 28,2003. Vol. 11.

[7]. Giuliano Franco. Francesca Franco. Bernadino Rammazini: The father of occupational medicine. Am J Of Public Health. Sept 2001. 91(9): 1382 .

[8]. Coffin C.T. Baker J.P. Contemporary OB/GYN. Ultrasound. July 2007.Vol. 52. Issue 7.7

[9]. Kevin Evans, Shawn Roll et al. Work related musculoskeletal disorders among registered diagnostic medical sonographers and vascular Technologists. Journal of Diagnostic Medical Sonography. Nov/Dec. 2009. Vol.25; 6. 287-29.

[10]. Coffin C.T. Baker J.P. Exam-Table- Evaluation. Ultrasound Product Evaluation For Oakworks Medical. Sound Ergonomics. 1-3.

[11]. ASA and ASUM Joint Guidelines for reducing injuries to sonographers/sonologists. Australian Society for Ultrasound in Medicine. Revised 2010. 4. 
[12]. Li G. Buckele P. Current Techniques for Assessing Physical Exposure To Work Related Musculo- Skeletal Risks with Emphasis on Posture Based Methods. 1999. 42(5). 674-95.

[13]. Preventing work related musculoskeletal disorders in sonography. Workplace solutions from the NIOSH. Sept.2006. 2.

[14]. Dodgeon J, Bernard F, Wilde J. Avoidance of musculoskeletal disorders during ultrasound scanning. Sound Ergonomics. LLC 2008. 1.

[15]. Vivien Gibbs. Pat Young. Work related musculoskeletal disorders in sonography and the Alexander Technique. Ultrasound. Nov. 2008. Vol.16. No 14. 213-219. 